\title{
Music drives brain plasticity
}

\author{
Lutz Jäncke
}

Address: Division of Neuropsychology, Psychological Institute, University of Zurich, Binzmühlestrasse 14, 8050 Zürich, Switzerland

Email: 1.jaencke@psychologie.uzh.ch

Fl000 Biology Reports 2009, I:78 (doi:10.34I0/BI-78)

The electronic version of this article is the complete one and can be found at: http://FI000.com/Reports/Biology/content///78

\begin{abstract}
Music is becoming more and more of an issue in the cognitive neurosciences. A major finding in this research area is that musical practice is associated with structural and functional plasticity of the brain. In this brief review, I will give an overview of the most recent findings of this research area.
\end{abstract}

\section{Introduction and context}

Professional musicians have been used over the last 15 years as a model for brain plasticity $[1,2]$. Why are musicians so interesting for plasticity research? First of all, they are experts in playing musical instruments. To play the demanding two three-second segments of the 11th variation from the sixth Paganini Etude by Franz Liszt, for example, requires the production of 30 notes per second. A tremendous amount of training is needed to achieve this kind of finger speed. Ericsson and colleagues [3] were among the first to show how much professional musicians do in fact practice. The authors showed that professional pianists and violinists practice for 7,500 hours before reaching the age of 18 years, whereas music teachers can look back on a total practice time of approximately 3,500 hours. This difference was unaffected by the quality of musical education since all musicians in this study had graduated from the prestigious Berlin Academy of Music. Thus, the amount of practice is one of the most important factors influencing musical expertise, at least in terms of the skill required to play a musical instrument. If musicians practice that much, it is hypothesised, they should show some kind of neuroanatomical and neurophysiological adaptations. Professional, semi-professional, and non-professional musicians have now been studied extensively in terms of the neuroanatomical and neurophysiological underpinnings of their expertise. In principle, three different approaches to studying plastic processes in musicians are possible: (a) The first approach is cross-sectional in nature and mostly employs quasi-experimental designs ('post-test-only designs with non-equivalent groups' in the terminology of Cook and Campbell [4]). With this design, musicians and nonmusicians are studied at the same point in time in terms of anatomical or functional brain measures. This approach has been widely used because it is relatively easy to collect the data. Differences between both groups are attributed mostly to the different learning histories of musicians and non-musicians. However, the interpretation of these data is limited since this approach does not allow the inference of strong causation because it cannot be ruled out that selection differences between the two groups or the different treatments (here, music lessons) are responsible for the results. To enhance the interpretability of such designs, several research groups have employed pretest measures related to musical expertise to control for pretest between-group differences. This design, which is called the 'untreated control group design with proxy pretest measures' [4], allows stronger causation about the influence of musical training. (b) The second approach used in this research context consists of short-term longitudinal studies in which subjects have undergone a specific training intervention. These studies are typically designed according to a prepost design, and the subjects are enrolled in training programs lasting from several hours to several months. (c) Finally, long-term longitudinal studies in which subjects have undergone a longer (at least a period of years) training are also used. Longitudinal studies are more complicated in terms of organisation of the experiments, they take longer, and they are more 
expensive. In addition, longitudinal studies are repeated measurements studies, implying some methodological problems (for example, unwanted practice effects). To understand the influence of music practice on brain plasticity more precisely, it is necessary to combine these different approaches.

A general finding of the studies published thus far is that nearly all of those brain areas involved in the control of musical expertise (motor cortex, auditory cortex, cerebellum, and other areas) show specific anatomical and functional features in professional and semi-professional musicians. In the following, I will review most of the recent papers (after 2002) supporting the idea of brain plasticity driven by musical expertise and musical training.

\section{Major recent advances}

\section{Structural brain plasticity}

Recently, Hyde and colleagues [5] published a paper strongly supporting the idea of use-dependent brain plasticity driven by musical training. In summary, this study demonstrates that 6-year-old children receiving instrumental musical training for 15 months (compared with children receiving non-musical training) not only learned to play their musical instrument but also showed changed anatomical features in brain areas known to be involved in the control of playing a musical instrument. Most of these brain areas are part of the cortical motor system, but there were also structural changes in the auditory system and in the corpus callosum. This is the first longitudinal study demonstrating brain plasticity in children in the context of learning a musical instrument.

Although longitudinal studies are the gold standard in plasticity research, several cross-sectional studies demonstrating specific anatomical features in musicians have recently been published. For example, Bangert and Schlaug [6] reported that pianists atypically showed the 'omega sign' (indicative of a larger hand motor area) on both hemispheres, whereas violinists showed the omega sign on only the right hemisphere controlling the left hand. This specific anatomical feature is possibly related to the fact that pianists practice a lot with both hands, whereas violinists practice a lot with their left hand (manipulating the strings) and their right arm (manipulating the bow). Thus, violinists might drive only the right-sided hand motor area, whereas pianists drive the hand motor areas on both hemispheres. This interesting finding is in strong concordance with older studies reporting specific anatomical features in the hand motor area in pianists and violinists $[7,8]$.

Using a voxel-based morphometry approach, Gaser and Schlaug [9] identified grey matter volume differences in motor, auditory, and visual-spatial brain regions when comparing professional musicians (keyboard players in this study) with a matched group of amateur musicians and non-musicians. Most interestingly, they found a strong association between structural differences (grey matter density), musician status, and practice intensity, supporting the view that practice (in this case, practicing to play a musical instrument) has an impact on brain anatomy. Increased grey matter density (and volume) is currently taken as evidence of an increase in capillary density as well as smaller changes in synapse and glial cell density. Thus, these changes might reflect neuroanatomical adaptations in order to improve the cognitive and motor functions controlled by these particular brain areas.

Most recently, a Swedish group used diffusion tensor imaging (DTI) to measure the integrity of fiber tracts (association fibers and commissures) in eight professional pianists and found a strong positive correlation between the measure of fractional anisotropy (FA) (indicating the integrity of the fiber system) and time spent practicing the piano $[10,11]$. Thus, the pianists who practiced more often showed higher FA values (indicating a higher integrity of the fiber system). This finding is of outstanding importance because it brings to light morphometric differences even within a highly specialised group of skilled pianists and indicates that these differences are due to practice time ('specialisation of the specialised'). In 2002, Schneider and colleagues [12], of Heidelberg, Germany, reported a remarkable anatomical finding in musicians. Using magnetoencephalography (MEG) and sophisticated anatomical analyses, the authors found neurophysiological and anatomical differences between musicians and nonmusicians. First, the neurophysiological activity in the primary auditory cortex $19-30 \mathrm{~ms}$ after tone onset was more than $100 \%$ larger. In addition, the grey matter volume of the anteromedial part of Heschl's gyrus (which covers most of the primary auditory cortex) was $130 \%$ larger in musicians. Both measures were also highly correlated with musical aptitude. This study is one of the first to indicate that both the morphology and neurophysiology of Heschl's gyrus have an essential influence on musical aptitude [12]. The second paper of the same group was even more spectacular [13]. In this paper, they found a strong relationship between the strategy used in processing complex tones and anatomical features in the primary auditory cortex. Professional musicians who preferentially analyse the fundamental pitch (the fundamental tone, abbreviated $\mathrm{fO}$ or $\mathrm{FO}$, is the lowest frequency in a harmonic series) of complex tones were found to have a leftward asymmetry of grey matter volume in Heschl's gyrus, whereas those who prefer to 
analyse the spectral pitch of complex tones show a rightward asymmetry of grey matter volume of Heschl's gyrus. Thus, a marked anatomical feature of the auditory system correlated with a particular tone-processing strategy within a group of professional musicians.

Patricia Sluming and colleagues [14], of Liverpool, UK, published a paper in which they reported anatomical differences in Broca's area between musicians and nonmusicians. In particular, the authors reported increased grey matter in Broca's area in the left inferior frontal gyrus in musicians. In addition, they observed significant age-related volume reductions in cerebral hemispheres, dorsolateral prefrontal cortex bilaterally, and grey matter density in the left inferior frontal gyrus in controls but not in musicians! In other words, musicians showed no or a smaller decrease in grey matter density in the frontal cortex compared with non-musicians with increasing age. (This is very important for aging research since the volume of the frontal cortex has been shown to correlate negatively with age $[15,16]$.) This anatomical study suggests that orchestral musical performance might promote use-dependent retention, and possibly expansion, of grey matter within Broca's area (a brain area that is responsible for speech production, language processing, and language comprehension as well as controlling facial neurons; it is named after Pierre Paul Broca, who discovered the area after studying the postmortem brain of a patient with a speech impairment). In addition, this study emphasises the significant point that shared neural networks (within Broca's area) are involved in the control of language and music. In a more recent study, the same group showed that Broca's area is also involved in the control of mental rotation, but only in musicians [17]. They relate this extraordinary finding to the sightreading skills of musicians. In sight reading, visuospatial cognition is related to some kind of language decoding. Broca's area might be involved in the control of this specific inter-relationship.

The most recent study to use DTI techniques was published by Imfeld et al. [18]. These authors measured the training effects on FA in the corticospinal tract (CST) of professional musicians and control non-musicians and found significantly lower FA values in both the left and the right CST in the musician group. Diffusivity, a parameter indicating the amount of water that diffuses along and across the axon, was negatively correlated with the onset of musical training in childhood in the musician group. A subsequently performed median split into an early- and a late-onset musician group (median of 7 years) revealed increased diffusivity in the CST of the early-onset group as compared with both the late-onset group and the controls. In conclusion, DTI was successfully applied in revealing plastic changes in white matter architecture of the CST in professional musicians. The present results challenge the notion that increased myelination induced by sensorimotor practice leads to an increase in FA, as has been suggested previously. Instead, training-induced changes in diffusion characteristics of the axonal membrane may lead to increased radial diffusivity reflected in decreased FA values. However, this issue deserves more intensive discussion about the methodological aspects associated with FA and diffusivity measurements.

\section{Functional brain plasticity}

Besides the above-mentioned specific anatomical features in musicians, several recent (and older) studies have shown specific neurophysiological adaptations. Recently, Lappe et al. [19] demonstrated particular changes with respect to the neurophysiological responses of the auditory cortex in non-musicians who trained for 2 weeks to play the piano. These authors randomly assigned subjects to one of two groups: one group learned to play a musical sequence on the piano, whereas the control group listened to the music that had been played by the other group. The authors demonstrated training-induced cortical plasticity using the musically elicited mismatch negativity (MMNm) from MEG measurements before and after training. The MMNm is a neurophysiological response reflecting the preattentive processing of auditory stimuli $[20,21]$.

The subjects who learned to play piano showed significant enlargement of MMNm after training compared with the group who only listened to the music. Thus, practicing to play the piano improves not only hand motor skills but also the auditory representation of the musical tones that are generated by the piano keys. Thus, a strong crossmodal link between motor commands and the representation of auditory information is established, causing a stronger representation of musical information in the auditory cortex. Several years ago, Bangert and Altenmüller [22] demonstrated a similar finding using electroencephalogaphy (EEG). They identified changed activations in frontal brain regions of subjects who had just 20 minutes of piano training, but only in the learning conditions during which the subjects could easily associate a particular piano key with a note. In situations during which this association was random, there was no cortical crossmodal plasticity. Effects of training have also been shown to be instrument-specific [23-25], and the EEG responses of children taking music lessons have been shown to change differently over the course of a year compared with those of children not studying music [26]. Thus, in summary, musicians or musically experienced subjects respond differently to 
musical stimuli even if top-down factors like attention are controlled for [27]. There is also ample evidence of change in the auditory system due to musical practice and in the entire sensorimotor system [28-32].

Interestingly, most of the recent findings indicate that even neurophysiological responses at the level of the brainstem are dependent on experience-dependent influences. Neural activity generated from the brainstem can be measured using frequency following responses (FFRs). The FFR is an electrophysiological scalp-recorded electrical response that reflects processing stages of auditory information at the level of the brainstem. Specifically, Wong et al. [33] first showed music-related plasticity in FFRs elicited by speech. Later, Musacchia et al. [34] found that musicians had more robust FFRs to auditory and audiovisual speech and music sounds. The latter study also strengthened the notion that musical experience shapes not only auditory processing but multisensory mechanisms as well. However, both studies indicate that playing music enhances the fidelity of the earliest stage of auditory response, not only to musical stimuli but also to speech and multisensory cues.

More recently, Krishnan et al. [35] analysed the FFRs from Chinese and English subjects in response to four Mandarin tonal contours presented in a non-speech context. The FFR analysis revealed that the Chinese group exhibited stronger representation of multiple pitchrelevant harmonics relative to the English group across all four tones. The authors concluded that long-term experience (here, experience with Mandarin) enhanced the sensitivity to linguistically relevant variations in pitch. Thus, specific language experience changes the FFR in a manner similar to that of music experience.

\section{Future directions}

The preceding findings give rise to the question of whether there is transfer from musical to non-musical skills. A well-trained auditory system might support the perception of auditory speech information and thus auditory speech information might be processed more efficiently. In addition, when learning to play a musical instrument, the trainee also practices attention, planning functions, memory, and self-discipline. It is thus hypothesised that musical experience would positively influence executive functions, language functions, or even intelligence in general. Several recently published papers are in line with this hypothesis. For example, one paper demonstrates that extended musical experience enhances executive control on a non-verbal spatial task and auditory tasks [36]. Glenn Schellenberg [37] uncovered a greater IQ increase in children enrolled in music classes compared with well-matched children who received no musical lessons, and Ho et al. [38] uncovered an enhancement of verbal memory skills (but not visual memory skills) in children enrolled in musical lessons. There is therefore mounting evidence on the behavioural level of positive transfer from musical expertise to nonmusical domains. Recently, Moreno et al. [39] established that musical training (not longer than 6 months) improves non-musical functions such as reading and linguistic perception. These non-musical enhancements are also accompanied by changed cortical activation patterns. This study is one of the very few longitudinal studies to have been conducted in the context of musical training.

If music has such a strong influence on brain plasticity, this raises the question of whether this effect can be used to enhance brain plasticity and cognitive performance in general and clinical settings. In a recent single-blind randomised controlled study, Särkämö et al. [40] examined whether daily music listening enhances the recovery of cognitive functions and mood after stroke. This study demonstrates that recovery of verbal memory and focused attention improved significantly and substantially in the group of patients who listened to their favourite music on a daily basis compared with patients who listened to audio books or received no listening material. Besides the cognitive improvement in the context of listening to music, there was a substantial mood improvement in the patients who listened to music. Thus, music could be used as a non-invasive tool for neuropsychological and neurological therapies. In addition, musical elements could be used to improve specific cognitive functions for which positive transfer effects have been demonstrated. For example, reading and writing skills as well as memory functions are possible candidates for functions that might benefit from musical training elements. Recent evidence shows that writing and reading can be improved when dyslexic children learn to associate graphemes and phonemes with musical notes [41] and that many memory elements are linked to music $[42,43]$. Hopefully, the current trend in the use of musicians as a model for brain plasticity will continue in future experiments and extend to the field of neuropsychological rehabilitation.

\section{Abbreviations}

CST, corticospinal tract; DTI, diffusion tensor imaging; EEG, electroencephalogaphy; FA, fractional anisotropy; FFR, frequency following response; MEG, magnetoencephalography; MMNm, musically elicited mismatch negativity.

\section{Competing interests}

The author declares that he has no competing interests. 


\section{References}

I. Münte TF, Altenmüller E, Jäncke L: The musician's brain as a model of neuroplasticity. Nat Rev Neurosci 2002, 3:473-8.

2. Schlaug G: The brain of musicians. A model for functional and structural adaptation. Ann N Y Acad Sci 200I, 930:28I-99.

3. Ericsson KA, Krampe RT, Clemens $\mathrm{T}$ : The role of deliberate practise in the acquisition of expert performance. Psychol Rev 1993, 100:363-406.

4. Cook TD, Campbell DT: Quasi-Experimentation: Design and Analysis Issues for Field Settings. Boston, MA: Houghton Mifflin Company; 1979.

5. Hyde KL, Lerch J, Norton A, Forgeard M, Winner E, Evans AC, Schlaug G: Musical training shapes structural brain development. J Neurosci 2009, 29:3019-25.

FI000 Factor 9.0 Exceptional

Evaluated by Lutz Jäncke 01 Apr 2009

6. Bangert $M$, Schlaug G: Specialization of the specialized in features of external human brain morphology. Eur J Neurosci 2006, 24: 1832-4.

7. Amunts K, Schlaug $G$, Jäncke L, Steinmetz $H$, Schleicher A, Dabringhaus A, Zilles K: Motor cortex and hand motor skills: structural compliance in the human brain. Hum Brain Mapp 1997, 5:206-15

8. Elbert T, Pantev C, Wienbruch C, Rockstroh B, Taub E: Increased cortical representation of the fingers of the left hand in string players. Science 1995, 270:305-7.

9. Gaser C, Schlaug G: Brain structures differ between musicians and non-musicians. J Neurosci 2003, 23:9240-5.

10. Bengtsson SL, Nagy Z, Skare S, Forsman L, Forssberg H, Ullén F: Extensive piano practicing has regionally specific effects on white matter development. Nat Neurosci 2005, 8: I | 48-50.

II. Han Y, Yang H, Lv YT, Zhu CZ, He Y, Tang HH, Gong QY, Luo YJ, Zang YF, Dong Q: Gray matter density and white matter integrity in pianists' brain: a combined structural and diffusion tensor MRI study. Neurosci Lett 2009, 459:3-6.

12. Schneider P, Scherg M, Dosch HG, Specht HJ, Gutschalk A, Rupp A: Morphology of Heschl's gyrus reflects enhanced activation in the auditory cortex of musicians. Nat Neurosci 2002, 5:688-94.

13. Schneider P, Sluming V, Roberts N, Scherg M, Goebel R, Specht HJ, Dosch HG, Bleeck S, Stippich C, Rupp A: Structural and functional asymmetry of lateral Heschl's gyrus reflects pitch perception preference. Nat Neurosci 2005, 8: I24|-7.

14. Sluming V, Barrick T, Howard M, Cezayirli E, Mayes A, Roberts N: Voxel-based morphometry reveals increased gray matter density in Broca's area in male symphony orchestra musicians. Neuroimage 2002, I7:1613-22.

15. Raz N, Lindenberger U, Rodrigue KM, Kennedy KM, Head D, Williamson A, Dahle C, Gerstorf D, Acker JD: Regional brain changes in aging healthy adults: general trends, individual differences and modifiers. Cereb Cortex 2005, I5:1676-89.

FI000 Factor 3.0 Recommended

Evaluated by Roberto Cabeza 27 May 2005

16. Raz N, Rodrigue KM, Haacke EM: Brain aging and its modifiers: insights from in vivo neuromorphometry and susceptibility weighted imaging. Ann N Y Acad Sci 2007, I097:84-93.

17. Sluming V, Brooks J, Howard M, Downes JJ, Roberts N: Broca's area supports enhanced visuospatial cognition in orchestral musicians. J Neurosci 2007, 27:3799-806.

18. Imfeld A, Oechslin MS, Meyer M, Loenneker T, Jäncke L: White matter plasticity in the corticospinal tract of musicians: a diffusion tensor imaging study. Neuroimage 2009, 46:600-7.

19. Lappe C, Herholz SC, Trainor LJ, Pantev C: Cortical plasticity induced by short-term unimodal and multimodal musical training. J Neurosci 2008, 28:9632-9.

FI000 Factor 3.0 Recommended

Evaluated by Lutz Jäncke 0 I Oct 2008
20. Näätänen R: Mismatch negativity (MMN) as an index of central auditory system plasticity. Int J Audiol 2008, 47:SI6-20.

21. Näätänen $R$, Paavilainen $P$, Rinne $T$, Alho $K$ : The mismatch negativity (MMN) in basic research of central auditory processing: a review. Clin Neurophysiol 2007, I I 8:2544-90.

22. Bangert $M$, Altenmüller EO: Mapping perception to action in piano practice: a longitudinal DC-EEG study. BMC Neurosci 2003, 4:26

23. Schulz M, Ross B, Pantev C: Evidence for training-induced crossmodal reorganization of cortical functions in trumpet players. Neuroreport 2003, I4:157-6I.

24. Shahin A, Bosnyak DJ, Trainor LJ, Roberts LE: Enhancement of neuroplastic $\mathrm{P2}$ and NIc auditory evoked potentials in musicians. J Neurosci 2003, 23:5545-52.

25. Pantev C, Roberts LE, Schulz M, Engelien A, Ross B: Timbre-specific enhancement of auditory cortical representations in musicians. Neuroreport 200I, I2:169-74.

26. Fujioka T, Ross B, Kakigi R, Pantev C, Trainor LJ: One year of musical training affects development of auditory corticalevoked fields in young children. Brain 2006, I29:2593-608.

27. Baumann S, Meyer M, Jäncke L: Enhancement of auditory-evoked potentials in musicians reflects an influence of expertise but not selective attention. J Cogn Neurosci 2008, 20:2238-49.

28. Bangert M, Peschel T, Schlaug G, Rotte M, Drescher D, Hinrichs H, Heinze HJ, Altenmüller E: Shared networks for auditory and motor processing in professional pianists: evidence from fMRI conjunction. Neuroimage 2006, 30:917-26.

29. Baumann S, Koeneke S, Schmidt CF, Meyer M, Lutz K, Jäncke L: A network for audio-motor coordination in skilled pianists and non-musicians. Brain Res 2007, I I 61 :65-78.

30. Lotze M, Scheler G, Tan HR, Braun C, Birbaumer N: The musician's brain: functional imaging of amateurs and professionals during performance and imagery. Neuroimage 2003, 20: 1817-29.

3I. Jäncke L, Baumann S, Koeneke S, Meyer M, Laeng B, Peters M, Lutz K: Neural control of playing a reversed piano: empirical evidence for an unusual cortical organization of musical functions. Neuroreport 2006, I7:447-5I.

32. Jäncke L, Shah NJ, Peters M: Cortical activations in primary and secondary motor areas for complex bimanual movements in professional pianists. Brain Res Cogn Brain Res 2000, I0:177-83.

33. Wong PC, Skoe E, Russo NM, Dees T, Kraus N: Musical experience shapes human brainstem encoding of linguistic pitch patterns. Nat Neurosci 2007, 10:420-2.

34. Musacchia G, Strait D, Kraus N: Relationships between behavior, brainstem and cortical encoding of seen and heard speech in musicians and non-musicians. Hear Res 2008, 241:34-42.

35. Krishnan A, Swaminathan J, Gandour JT: Experience-dependent enhancement of linguistic pitch representation in the brainstem is not specific to a speech context. J Cogn Neurosci 2009, 2 I: 1092-105.

FI000 Factor 6.0 Must Read Evaluated by Lutz Jäncke 07 Jul 2009

36. Bialystok E, Depape AM: Musical expertise, bilingualism, and executive functioning. J Exp Psychol Hum Percept Perform 2009, 35:565-74.

37. Schellenberg EG: Music lessons enhance IQ. Psychol Sci 2004, | 5:5 | |-4.

38. Ho YC, Cheung MC, Chan AS: Music training improves verbal but not visual memory: cross-sectional and longitudinal explorations in children. Neuropsychology 2003, I 7:439-50.

39. Moreno S, Marques C, Santos A, Santos M, Castro SL, Besson M: Musical training influences linguistic abilities in 8-year-old children: more evidence for brain plasticity. Cereb Cortex 2009, 19:712-23.

FI000 Factor 6.0 Must Read Evaluated by Lutz Jäncke 21 Jul 2009 
40. Särkämö $T$, Tervaniemi $M$, Laitinen $S$, Forsblom $A$, Soinila $S$, Mikkonen M, Autti T, Silvennoinen HM, Erkkilä J, Laine M, Peretz I, Hietanen M: Music listening enhances cognitive recovery and mood after middle cerebral artery stroke. Brain 2008, I3 I: 866-76.

FI000 Factor 6.0 Must Read

Evaluated by Lutz Jäncke 16 May 2008
4I. Kast M, Meyer M, Vogeli C, Gross M, Jäncke L: Computer-based multisensory learning in children with developmental dyslexia. Restor Neurol Neurosci 2007, 25:355-69.

42. Eschrich S, Münte TF, Altenmüller EO: Unforgettable film music: the role of emotion in episodic long-term memory for music. BMC Neurosci 2008, 9:48.

43. Jäncke L: Music, memory and emotion. J Biol 2008, 7:21. 